\title{
De qué hablamos cuando hablamos de universidades. El Estado Chileno y la idea contemporánea de la universidad
}

\author{
Julio Labraña Vargas ${ }^{1}$
}

Resumen: La discusión acerca de la universidad contemporánea tiende a analizar las instituciones de educación superior en términos normativos. En contraste, este paper sugiere que la idea de la universidad es socialmente construida. Enfocándose en la legislación chilena acerca de la universidad y sus mecanismos de aseguramiento de la calidad, se argumenta que la universidad ya no se entiende como una institución con tres distintas funciones (investigación, enseñanza y extensión) sino como una institución cuya función principal es desempeñar su propia misión. Se sugiere que la noción de Readings de 'universidad de excelencia' puede contribuir a explicar estas transformaciones.

Palabras clave: Idea de universidad. Masificación. Educación superior.

\section{What we talk about when we talk about universities. The Chilean State and the contemporary idea of the university}

Abstract: The literature on the idea of the contemporary university tends to analyze higher education institutions in normative terms. In contrast, this paper suggests that the idea of the university is socially constructed. By focusing on the Chilean university legislation and quality assurance, it is argued that the university is no longer understood as an institution with three different functions (research, teaching, and extension) but as an institution whose main function is to fulfill its own mission. It is suggested here that Readings's notion of 'university of excellence' can help to explain these changes.

Key words: Idea of the university. Massification. Higher education.

\section{O que falamos quando falamos de universidades. O Estado chileno e a ideia contemporânea da universidade}

Resumo: Discussão sobre a universidade contemporânea tende a analisar as instituições de ensino superior em termos normativos. Em contraste, este artigo sugere que dá ideia da universidade é socialmente construída. Com foco na lei chilena sobre a universidade e seus mecanismos de garantia de qualidade, argumenta-se que a universidade não entendida como uma instituição com três funções diferentes (pesquisa, ensino e extensão), mas como uma instituição cuja função principal é cumprir sua própria missão. Sugere-se que a noção de Readings de "universidade de excelência" pode ajudar a explicar essas transformações.

Palavras-chave: Ideia da universidade. Massificação. Ensino superior. 
De qué hablamos cuando hablamos de universidades. El Estado chileno y la idea contemporánea de la universidad

\section{Introducción}

El debate acerca de la idea de la universidad contemporánea ha sido abordado de manera repetida en la literatura. Gran parte de los análisis evalúan a las instituciones de educación superior y las clasifican en términos de su grado de cercanía respecto a un ideal definido de antemano. Así, la crítica utiliza conceptos como mercantilización (HAYES; WYNYARD, 2002) o destaca las amenazas del neoliberalismo a la universidad (READINGS, 1996; SLAUGHTER; LESLIE, 2001; GIROUX, 2002).

En cambio, mucho menos ensayados han sido análisis que consideren la emergencia de normas y sus críticas acerca de la universidad como elementos de estudio, esto es, que partan de una aproximación autorreferencial de la sociedad (LUHMANN; SCHORR, 2000). En el siguiente artículo se seguirá esta propuesta constructivista para describir la constitución, aceptación y legitimación de nuevos modelos para las instituciones de educación superior en el caso de Chile, si bien la extensión de estas tendencias demuestra la aparición mundial de concepciones alternativas de la universidad.

La elección de Chile no es casual. Chile exhibe uno de los sistemas de educación superior con mayor grado de mercantilización en el mundo, convirtiéndose de este modo en un objeto de estudio interesante para la comunidad de investigadores en educación superior (BRUNNER, 1993; GINSBURG et al., 2005; BENEDIKTER; SIEPMANN; ZLOSILO, 2015). Además, en los últimos años, tras décadas de comportamiento subsidiario por parte del Estado, estudiantes, académicos y miembros de las instituciones de educación superior discuten nuevamente qué caracteriza a una universidad (MARTINIC et al., 2014). Parte de estas aproximaciones analizan la influencia de tendencias estructurales en el desarrollo de las universidades (BERNASCONI, 2006, 2008; SALAZAR; LEIHY, 2013; BRUNNER, 2014a, 2014b), en tanto otras recomiendan características deseables (HAX; UGARTE, 2014).

A pesar de este interés, no parece haber existido similar reflexión en torno a las estructuras institucionales que directa o indirectamente han promovido una concepción de la universidad. En el caso de Chile, los criterios y decisiones de la instancia reguladora de la creación y obtención de autonomía de las nuevas universidades privadas creadas después de 1991, el Consejo Superior de Educación, ha recibido comparativamente escasa atención en la literatura especializada.

En esta dirección, este estudio busca observar los criterios decisionales del Consejo Superior de Educación y sus consecuencias en términos de discursos acerca de la universidad contemporánea. El artículo se estructura en las siguientes categorías. En primer lugar, se 
revisa la literatura en torno a los horizontes normativos de las instituciones de educación superior (I). A continuación, se describe la institucionalidad por la que debe pasar toda nueva universidad chilena en su proceso de creación, reconocimiento oficial y obtención de autonomía plena (II). En tercer lugar, se analizan las decisiones y fundamentaciones utilizadas por el Consejo Superior de Educación entre 1991 y 2015 para no aceptar o revocar el reconocimiento oficial a una universidad (III). En cuarto lugar, se interpretan estos resultados a la luz de la discusión acerca actual acerca de la idea de la universidad moderna (IV). El estudio finaliza con una reflexión, futuras líneas de estudio y posibles consecuencias normativas (V).

\section{Los horizontes normativos de las instituciones de educación superior}

En la literatura especializada, existen varias aproximaciones al debate acerca del rol de la universidad en la sociedad contemporánea. Dichas aproximaciones pueden ser distinguidas de acuerdo a si su énfasis es analítico (centrado en explicar las variaciones en el ideario universitario) o normativo (centrado en proponer atributos que deben caracterizar a las instituciones de educación superior). El primer grupo de reflexiones asume un énfasis en los cambios estructurales o las relaciones de poder que median la definición de la idea de la universidad) (BERNASCONI, 2006; SCOTT, 2006; BARBLAN, 2011; DMITHISHIN, 2013; SALAZAR; LEIHY, 2013). Por el contrario, la segunda aproximación tiende a criticar y proponer marcos normativos adecuados para el desarrollo presente y futuro de estas instituciones educativas (HABERMAS, 1987; GIROUX, 2002; PUSSER, 2006; SIT, 2010).

Por supuesto, estos marcos no son excluyentes. Los análisis suelen poseer repercusiones normativas y las sugerencias acerca del futuro de las universidades acostumbran a basarse en una interpretación de la evolución de las instituciones de educación superior. A pesar de ello, la diferencia es útil, en tanto sirve para distinguir el énfasis de los estudios. En este sentido, en las siguientes reflexiones analíticas, se seguirá un principio constructivista de análisis: las universidades y su forma de reflexión son específicamente aquello que se acepta y comprende como tal en la sociedad.

En particular, el énfasis de este estudio está en el examen de los mecanismos mediante los cuales ciertas organizaciones adquieren la calificación para ser vistas como universidades en la sociedad. Por lo tanto, como suele ser el caso en los sistemas universitarios nacionales (LABRAÑA, 2016a), el Estado, entendido como el conjunto de organismos dependientes del Poder Ejecutivo y a otras entidades autónomas que representan la sociedad organizada 
De qué hablamos cuando hablamos de universidades. El Estado chileno y la idea contemporánea de la universidad

(GONZÁLEZ; ESPINOZA, 2008, p. 255), cumple un rol fundamental en definir los criterios que permiten reconocer aquello propio de las universidades.

Esta aproximación puede parecer demasiado empírica para un debate tan abstracto como es la idea de la universidad. Sin embargo, como se argumentará en este estudio, estas decisiones condicionan la forma de los discursos acerca de la universidad (véase ARCHER, 2003 , p. 247 y ss).

\section{El reconocimiento y obtención de autonomía de las nuevas universidades chilenas}

El sistema de la educación superior chileno se caracteriza por un elevado grado de mercantilización y una baja participación del Estado desde las reformas impuestas por la dictadura militar de Pinochet en los ochenta (BERNASCONI; ROJAS, 2003). En general, los gobiernos democráticos posteriores no introdujeron transformaciones importantes en la conceptualización de las universidades y, en cambio, optaron por consolidar dichas reformas a través de la creación de mecanismos de accountability para las universidades (COMISIÓN BRUNNER, 1991).

Un rol central en la definición de qué es una universidad lo jugó la Ley Orgánica Constitucional de Enseñanza (LOCE), $\mathrm{N}^{\circ} 18.962$ del 10 de marzo de 1990. Esta normativa establece las siguientes etapas para crear una institución de educación superior (BERNASCONI; ROJAS, 2003, p. 37): 1) obtención de la personalidad jurídica; 2) reconocimiento oficial, por medio de la cual la entidad puede iniciar actividades académicas; y 3) plena autonomía, que habilita para entregar títulos y grados

En lo que respecta a la creación, en su artículo 44 esta normativa establece que las universidades no creadas por ley, deben constituirse por escritura pública o por instrumento privado reducido a escritura pública, la que debe contener el acta de constitución de la entidad y sus estatutos. En su artículo 45, señala que los estatutos de estas instituciones debían, además, individualizar sus organizadores; indicar el nombre y domicilio de entidad; sus fines; los medios económicos y financieros de que dispone para su realización; la estructura de gobierno de la nueva entidad y la forma en que ella excluyese la participación con derecho a voto de los alumnos y de los funcionarios administrativos; los títulos profesionales y grados académicos de licenciado que entregara inicialmente; y, finalmente, disposiciones relativas a la modificación de estatutos y a su disolución. Finalmente, su artículo 47 indica que el Ministerio de Educación no podía negar el registro de una universidad, si bien tenía la 
posibilidad de objetar su creación si es que sus estatutos no cumplían con lo prescrito en la ley.

En lo que respecta al reconocimiento oficial, el artículo 30 de la Ley Orgánica Constitucional de Enseñanza, $N^{\circ} 18.962$ señala que las universidades debían constituirse por los procedimientos establecidos en esta ley, siendo siempre corporaciones de derecho privado, sin fines de lucro. El artículo 50 indica los requisitos para su reconocimiento oficial: estar constituidas como personas jurídicas; tener la certificación del Consejo Superior de Educación de contar con los recursos docentes, didácticos, económicos, financieros y físicos necesarios para ofrecer sus grados académicos y títulos profesionales; y poseer la certificación del Consejo Superior de Educación que establezca que la nueva universidad hubiese aprobado su proyecto institucional y programas y, además, que ella se sometería a dicho Consejo para llevar a cabo la verificación progresiva de su desarrollo institucional. En el caso que la institución cumpliese con los requisitos anteriormente señalados, señala el artículo 51, el Ministerio de Educación debía dictar un decreto de reconocimiento oficial dentro del plazo de treinta días. Tras ello, las nuevas universidades podían iniciar sus actividades, con la única obligación de ofrecer al menos uno de los títulos profesionales cuya obtención requiere el grado de licenciado.

Las nuevas universidades obtenían su plena autonomía cuando dejaban de estar bajo la verificación del Consejo Superior de Educación. El artículo 75 define la autonomía como el derecho de la institución educativa a regirse por sí misma económica, académica y administrativamente.

En el momento de creación del Consejo Superior de Educación, existían dos regímenes de regulación: la acreditación (actual licenciamiento) ${ }^{1}$ a cargo del Consejo Superior de Educación y la examinación, proceso en que los alumnos y programas de las nuevas instituciones eran evaluados por las universidades creadas antes de 1981. Hasta 1992 universidades e institutos profesionales debieron elegir si se sometían a procesos de examinación o licenciamiento. Debido a los menores costos que implicaba comparada con la examinación, la mayoría de instituciones optó por la acreditación.

El artículo 53 establece los límites de la autonomía de las universidades. Ella define las condiciones en las que el Ministerio de Educación, previo informe del Consejo Superior de Educación, podía cancelar la personalidad jurídica y revocar el reconocimiento oficial de una

\footnotetext{
${ }^{1}$ En adelante se denominará a este proceso como licenciamiento. El concepto de acreditación será utilizado exclusivamente al hacer referencia a los procesos de evaluación de las instituciones autónomas de educación superior. 
De qué hablamos cuando hablamos de universidades. El Estado chileno y la idea contemporánea de la universidad

universidad: si no cumplía con sus objetivos estatutarios; si realizaba actividades contrarias a la moral, al orden público, a las buenas costumbres y a la seguridad nacional; si incurría en infracciones graves a sus estatutos; o si dejaba de otorgar títulos profesionales que requieren haber obtenido previamente el grado de licenciado. En esta dirección, como destacan Bernasconi y Rojas (2003, p. 39), en el ordenamiento chileno la autonomía no constituye una prerrogativa ilimitada de las universidades, en tanto el Estado siempre tiene la posibilidad disponible de revocarle el reconocimiento y cerrar estas instituciones.

A medida que las instituciones de educación superior optaron por el licenciamiento antes que por la examinación por parte de las universidades antiguas, la importancia del Consejo Superior de Educación aumentó. Su fiscalización definió cuáles fueron las instituciones reconocidas por el Estado y, además, tras un periodo de verificación progresiva, el Consejo era el encargado de dar las garantías de que estas universidades podían operar autónomamente en términos académicos, económicos y administrativos. De este modo, el Consejo Superior de Educación se constituyó en el primer regulador que aseguraba que ciertas instituciones podían utilizar el nombre de universidades. Como se verá a continuación, sus decisiones cambiaron progresivamente aquello que en Chile se comprendió como propio de las instituciones universitarias - esto es: su idea característica y horizonte normativo.

\section{La labor del Consejo Superior de Educación}

Como todo arreglo de la educación superior, las decisiones acerca de aprobación o rechazo de proyectos de nuevas universidades tienen consecuencias en la comprensión del horizonte normativo del sistema de educación superior. En esta dirección, mientras una estructura con elevados grados de exigencia tiende a restringir la aparición de nuevas universidades y promueve la canalización de la demanda por estudios terciarios a otro tipo de instituciones de educación superior, formas de regulación menos estrictas abren espacio para la aparición de nuevos modelos de universidades. En el caso de seguirse esta segunda opción, la misma noción de la universidad se problematiza a través de la legitimación por parte del Estado de modelos alternativos a las universidades tradicionales.

La labor del Consejo Superior de Educación (CSE) apunta en esta última dirección. Este órgano fue creado por la Ley Orgánica Constitucional de Enseñanza, $\mathrm{N}^{\circ} 18.962$ del 10 de marzo de 1990. Entre otras tareas, su labor considerada pronunciarse sobre los proyectos institucionales presentados por las distintas universidades e institutos profesionales para efectos de su reconocimiento oficial, verificar progresivamente el desarrollo de los proyectos 
institucionales de conformidad a las normas de acreditación establecidas en la Ley $\mathrm{N}^{\circ} 18.962$, y recomendar al Ministerio de Educación la aplicación de sanciones de cierre y revocación de reconocimiento a las entidades en licenciamiento (CSE, 1991).

La Ley $\mathrm{N}^{\circ} 20.129$ del 17 de noviembre de 2006, que estableció un sistema nacional de aseguramiento de la calidad de la educación superior, agregó a las funciones del CSE el licenciamiento de centros de formación técnica. Con la dictación de la Ley $\mathrm{N}^{\circ}$ 20.370, General de Educación, publicada el 12 de septiembre de 2009, el Consejo Nacional de Educación se constituyó en el sucesor legal del Consejo Superior de Educación y asumió las funciones del anterior organismo, además de adquirir nuevas responsabilidades en torno a la fiscalización de la enseñanza en los niveles básico y medio² ${ }^{2}$.

Si se analiza la labor del Consejo Superior de Educación y de su sucesor así como las características de las instituciones cuyo reconocimiento fue revocado, es fácil llegar a la conclusión que en sus decisiones tuvo especial relevancia el análisis de problemas financieros y de administración y gestión de las instituciones antes que materias relativas a la conservación de un modelo universitario tradicional. En esta dirección, los criterios utilizados para definir el reconocimiento y licenciamiento oficial tuvieron por objetivo promover la autorregulación de las instituciones de educación superior. Más allá del cumplimiento del requisito establecido en la Ley Orgánica Constitucional de Enseñanza de que las nuevas universidades otorgasen, al menos, un título profesional cuya obtención requiriese el grado académico de licenciado, las observaciones del Consejo se enfocaron en determinar si es que estas instituciones poseían las condiciones para definir autónomamente sus propósitos académicos.

En este sentido apunta también la literatura acerca del Consejo Superior de Educación. Ella sugiere que su horizonte último de evaluación se encontraba en el proyecto institucional presentado por las instituciones y no en su concordancia con un modelo explícito acerca de lo que debían ser las actividades universitarias (LEMAITRE, 1997; LAVADOS, 1998). Esta visión también es compartida por los testimonios de las instituciones educativas que pasaron por estas instancias de evaluación (véase como ejemplos CASTRO, 1997; ALBORNOZ, 1997; LUCERO; THIERS, 1998; HERNÁNDEZ, 1998),

Por lo demás, esta es una cuestión que no sólo estaba determinada por el Consejo. En última instancia, este órgano debió operar en función de la normativa definida en la Ley

\footnotetext{
${ }^{2}$ En adelante, para mantener la consistencia a lo largo del texto, utilizaremos la denominación utilizada en la creación de este organismo: Consejo Superior de Educación.
} 
De qué hablamos cuando hablamos de universidades. El Estado chileno y la idea contemporánea de la universidad

General de Educación, $\mathrm{N}^{\circ}$ 20.370, coincidentes en términos de la labor de reconocimiento y licenciamiento con lo definido previamente en la Ley Orgánica Constitucional de Enseñanza, $\mathrm{N}^{\circ}$ 18.962. En consecuencia, este órgano no podía desviarse significativamente de las variables ya enunciadas en la legislación ${ }^{3}$.

Esta operatoria tuvo consecuencias importantes en la configuración del sistema de educación y en la conformación de sus horizontes normativos. Una vez que el Estado chileno aceptó la creación de universidades que cumplían una labor exclusivamente docente, la caracterización de la universidad como una institución que realizaba simultáneamente docencia, investigación y extensión se convirtió necesariamente en problemática. En efecto, si estas últimas funciones eran sólo opcionales para el desarrollo de las instituciones de educación superior, la valoración de una institución en línea con el imaginario de la universidad humboldtiana fue reemplazada por la apreciación de la diversidad (LABRAÑA, 2016b). En el sistema de la educación superior chileno conviven instituciones de investigación, con otras enfocadas en labores de profesionalización y aún otras que configuran su misión en la expresión de una identidad religiosa, política o artística. De esta manera, la apelación a una idea de universidad se convierte en una materia a definir en función de cada misión institucional.

\section{La idea de la universidad, ¿todavía?}

Por supuesto, como demuestra la literatura, estas transformaciones no son exclusivas del caso chileno. La proliferación de universidades docentes tiende a acompañar mundialmente el proceso de masificación de la cobertura y diversificación de la educación superior (LEVY, 1990; BRUNNER, 2014a).

Readings (1996) describe este proceso en términos del abandono de la asociación entre alta cultura y universidad y su reemplazo por la idea de excelencia. De acuerdo a su análisis, al contrario de la idea de cultura, la excelencia no poseería referente externo, en tanto se agota en la propia operación de cada institución de educación superior (p. 39). En esta misma dirección, como hemos visto, los marcos evaluativos no pueden exceder lo definido por las

\footnotetext{
3 ¿Significa eso que la labor de verificación del Consejo es un simple trámite burocrático? La respuesta es negativa. Si bien creada para legitimar a las nuevas universidades, sus promotores en democracia utilizaron esta estructura para distinguir entre las instituciones que podían entregar títulos profesionales y grados académicos y aquellas que carecerían de los requisitos, especialmente financieros, administrativos y de gobierno
} 
universidades y se limitan a certificar que la institución está en condiciones de cumplir con lo que declara (LEMAITRE, 1997).

En cualquier caso, una vez reconocidas como tales, estas universidades docentes reclaman sus derechos de existencia. Legitimadas estatalmente como organizaciones universitarias, estas instituciones comparten la denominación con instituciones con modelos complejos. Simultáneamente, ello implica que sus administradores, académicos y estudiantes toman posiciones de interés en lo que respecta a la definición y alcance de estas instituciones, especialmente en lo que respecta a los criterios de financiamiento avanzados por el Estado. Así, y tal como demuestra la discusión actual a propósito de la reforma a la educación superior, ello aumenta la improbabilidad de alcanzar un consenso, en tanto toda medida general afecta la posición competitiva de actores con interés en la materia.

En base a lo anterior, es preciso preguntarse si la idea de la universidad puede ser algo más que un discurso cuando se promueve la existencia de distintas instituciones cuyo horizonte normativo se agota en sus propios fines y objetivos. Para responder esta pregunta, es necesario recordar los orígenes de la discusión acerca de la idea de la universidad: un contexto en que la matrícula de estas instituciones tendía a ser homogénea, sus funciones eran similares y el Estado jugaba un importante rol en términos de promoción del isomorfismo institucional (DIMAGGIO; POWELL, 1983). Por lo mismo, si es que ellos existían, los sistemas de aseguramiento de la calidad jugaban un rol comparativamente menor, en tanto la calidad de las instituciones estaba asegurada por su mera existencia. Así, de manera implícita, como en el contexto de 'autonomía privilegiada' característico de las universidades latinoamericanas (BRUNNER; BRIONES, 1992), o explícita, como era el caso de los países de Europa Occidental con Estado de Bienestar, ambos actores - Estado y universidades coincidían en la definición de sus ámbitos territoriales nacionales como marco de referencia y acción.

La noción de una universidad nacional (NEAVE, 2011) ya no es suficiente para describir la operación de las instituciones de educación superior, en tanto cada universidad combina de manera diferente los horizontes de sus espacios locales, nacionales y globales (MARGINSON; RHOADES, 2002; MARGINSON, 2004). Por lo tanto, una idea normativa de las universidades parece extraña al estado actual de estas instituciones. En efecto, si sus propósitos son una función de la decisión de estas organizaciones y si ello es, además, aceptado y reconocido como tal por los mecanismos de evaluación creados por el Estado, entonces la validez de una idea común acerca de la universidad se pierde. 
De qué hablamos cuando hablamos de universidades. El Estado chileno y la idea contemporánea de la universidad

¿Significa esto que la idea de la universidad es sólo un recurso argumentativo? No necesariamente. Como demuestra Readings (1996, p. 44-53), el horizonte normativo de estas instituciones estuvo acompañado de una asociación de estas instituciones con el Estado, órgano que mediante la idea de cultura definía, de manera implícita y con la colaboración de la similitud interna de las instituciones (LABRAÑA, 2016a), el ámbito de acción deseado para las universidades. En esta dirección, la actual selección estatal de objetivos a cumplir por determinadas instituciones - sean ellas o no estatales - ofrece la posibilidad de recrear un ideal para el sistema nacional de educación.

La literatura normativa sobre la universidad cuenta con avances interesantes en esta dirección. Examinando la discusión internacional acerca de los bienes públicos, GuzmánValenzuela (2015) propone hablar de una universidad transformativa. Su noción recoge las principales dimensiones de la labor pública de las instituciones de educación superior para sugerir una universidad en la que los estudiantes desarrollen sus carreras en base al mérito, se auto socialicen críticamente, y produzcan, junto a otros integrantes de la universidad, conocimiento para su entorno local, nacional y global.

Es improbable que todas las universidades pueden adquirir estas características. Sin embargo, es posible pensar en un grupo de instituciones de educación superior que representan el ideal al que las restantes instituciones debiesen esperar, definición en la cual el Estado posee un rol insoslayable. Al menos en Chile, este rol fue cumplido por las ocho universidades existentes antes de las reformas de 1981 por razones más relacionadas con su historia que con su desempeño objetivamente medido. La literatura contemporánea acerca de la universidad demuestra la centralidad del debate acerca de la universidad en un contexto en que la diversidad es definida como valor (STÖLTING; SCHIMANK, 2001).

\section{Conclusiones}

En este artículo se realizó una descripción acerca de qué es una universidad a través del análisis de los mecanismos que median su creación, reconocimiento y obtención de autonomía. En particular, este estudio se concentró en la experiencia del Consejo Superior de Educación en Chile y la revisión de sus criterios de definición acerca de qué es una universidad. A continuación, se integró este análisis en la discusión acerca de su horizonte normativo. Para ello se utilizó la tesis de Readings (1996) sobre la universidad de excelencia. Por último, se discutió la posibilidad de re(crear) la idea de la universidad. 
Los resultados de este artículo abren nuevas perspectivas. En tanto se examinaron de manera preferente los criterios de discriminación del Estado, se hace también necesario complementar este análisis con la observación de otros actores del establishment educativo. Una opción en ese sentido, es profundizar en las distinciones utilizadas en el lenguaje cotidiano de distintos actores sociales para definir qué no es realmente una universidad, a pesar de llevar legalmente este nombre: universidades chantas (Chile), pseudouniversities (Estados Unidos), universidades de garaje (Colombia), etc.

Otra posibilidad de análisis es comparar otros órganos con una función similar a la del Consejo Superior de Educación en la definición de los requisitos de una universidad. Tradicionalmente, estas entidades están sujetas a normativas legislativas y poseen una relación explícita con el Estado, si bien tienden a ser autónomas en su operar. En una dirección similar, el examen de los organismos de acreditación puede ser una siguiente etapa en la comprensión de los distintos modelos de universidad.

En términos normativos, vale la pena considerar este artículo para la formulación de marcos normativos para los sistemas de educación superior. Si las apelaciones a la idea de la universidad buscan ser más que recursos retóricos, entonces ellas deben reconocer la diversidad de misiones y objetivos en las universidades contemporáneas. Sin embargo, como se argumentó anteriormente, ello no implica que el ideal de las universidades deba ser dejado a la decisión de cada organización. La definición estatal de acciones a realizar por instituciones de educación superior puede contribuir, dentro de un rango mucho más limitado, a establecer la labor propia de estas instituciones. A través de mayores exigencias, el Estado podría así establecer un horizonte mínimo para las universidades.

\section{Referências}

ALBORNOZ, Mario. Universidad de Las Américas. En: PERSICO, María Cecilia; PERSICO, Pablo (Eds.). Acreditación en Chile. La experiencia de un lustro. Santiago, Chile: Corporación de Promoción Universitaria, 1997. p. 101-104.

ARCHER, Margaret. Realist social theory: the morphogenetic approach. Cambridge: Cambridge University Press, 2003.

BARBLAN, Andris. From the universities in Europe to the universities of Europe. En: RÜEGG, Walter (Ed.). A history of the university in Europe. Universities since 1945. Cambridge: Cambridge University Press, 2011. v. 4, p. 550-574

BENEDIKTER, Roland; SIEPMANN, Katja; ZLOSILO, Miguel. The educational dimension: Michelle Bachelet II's “Master Plan” for Chile's Future -The reform of education”. En: 
De qué hablamos cuando hablamos de universidades. El Estado chileno y la idea contemporánea de la universidad

BENEDIKTER, Ronald; SIEPMANN, Katja (Eds.). Chile in transition: prospects and Challenges for Latin America's Forerunner of Development. Cham: Springer International Publishing, 2015. p. 159-187.

BERNASCONI, Andrés. Breaking the institutional mold: faculty in the transformation of chilean higher education from state to market. En: DIETER-MAYER, Heinz; ROWAN, Brian (Eds.). The new institutionalism in education. New York: Albany State University of New York Press, 2006. p. 33-50.

BERNASCONI, Andrés. Is there a Latin American model of the university? Comparative Education Review, United States, v. 52, n. 1, p. 27-52, 2008.

BERNASCONI, Andrés; ROJAS, Fernando. Informe sobre la educación superior en Chile: 1980-2003. Santiago, Chile: Editorial Universitaria, 2003.

BRUNNER, José Joaquín. Chile's higher education: between market and state. Higher Education, the United States, v. 25, n. 1, p. 35-43, 1993.

BRUNNER, José Joaquín. La idea de la universidad pública en América Latina: narraciones en escenarios divergentes. Educación XXI, España, v. 17, n. 2, p. 17-34, 2014a.

BRUNNER, José Joaquín. Transformación de lo público y el reto de la innovación universitaria. Bordón revista de Pedagogía, España, v. 66, n. 1, p. 45-60, 2014b. Número monográfico Gobierno y gobernanza de la universidad: el debate emergente.

BRUNNER, José Joaquín; BRIONES, Guillermo. Higher Education in Chile: effects of the 1980 reform. En: WOLFF, Laurence; ALBRECHT, Douglas (Eds.). Higher education reform in Chile, Brazil and Venezuela. Washington D.C.: The World Bank, World Bank Paper LATHR n. 34, 1992.

CASTRO, Elisa. Universidad Tecnológica Vicente Pérez Rosales. En: PERSICO, María Cecilia; PERSICO, Pablo (Eds.). Acreditación en Chile. La experiencia de un lustro. Santiago, Chile: Corporación de Promoción Universitaria, 1997. p. 35-41.

COMISIÓN DE ESTUDIOS DE LA EDUCACIÓN SUPERIOR (COMISIÓN BRUNNER). Una política para el desarrollo de la educación superior en la década de los noventa. Informe de la Comisión de Estudio de la Educación Superior. Santiago, Chile: Ministerio de Educación, 1991.

CONSEJO SUPERIOR DE EDUCACIÓN (CSE). Un año de trabajo. Santiago, Chile: Consejo Superior de Educación, 1991.

DIMAGGIO, Paul J.; POWELL, Walter W. The Iron Cage Revisited: Institutional Isomorphism and Collective Rationality in Organizational Fields. American Sociological Review, United States, v. 48, n. 2, p. 147-160, 1983.

DMITHISHIN, Alexander. Deconstructing distinctions. The European University in comparative historical perspective. Entremons. UPF Journal of World History, España, n. 5, p. 1-18, 2013.

GINSBURG, Mark et al. Globalisation and higher education in Chile and Romania: the Roles of the International Monetary Fund, World Bank, and World Trade Organization. En: ZAJDA, Joseph (Ed.). International handbook on globalisation, education and policy research. Global pedagogies and policies. The Netherlands: Springer, 2005. p. 221-234.

GIROUX, Henry. Neoliberalism, corporate culture, and the promise of higher education: The university as a democratic public sphere. Harvard Educational Review, United States, v. 72, n. 4, p. 425-463, 2002. 
GONZÁLEZ, Luis Eduardo; ESPINOZA, Oscar. Calidad de la educación superior: concepto y modelos. Calidad de la Educación, Chile, n. 28, p. 247-276. 2008.

GUZMÁN-VALENZUELA, Carolina. Unfolding the meaning of public(s) in universities: toward the transformative university. Higher Education, United States, v. 71, n. 5, p. 1-13, 2015 .

HABERMAS, Jürgen. The Idea of the University - Learning Processes. New German Critique, United States, n. 41, p. 3-22, 1987.

HAX, Arnoldo; UGARTE, Juan José. Hacia la gran Universidad Chilena. Un modelo de transformación estratégica. Santiago, Chile: Ediciones UC, 2014.

HAYES, Dennis; WYNYARD, Robin (Eds.). The McDonaldization of higher education. Westport, CT; London: Bergin and Garvey, 2002.

HERNÁNDEZ, Sergio. Autorregulación y acreditación en la educación superior. La visión de la universidad del desarrollo. En: PÉRSICO JIMÉNEZ, Pablo; PÉRSICO JIMÉNEZ, María Cecilia (Eds.). Acreditación de instituciones autónomas de educación superior. Santiago, Chile: Corporación de Promoción Universitaria, 1998. p. 77-82.

LABRAÑA, Julio. El concepto de sistema de los sistemas de educación superior: el caso chileno. Calidad en la educación, Chile, n. 44, 2016 a.

LABRAÑA, Julio. La diversidad como valor de la educación superior chilena. Pensamiento Educativo, Chile, v. 53, n. 1, p. 1-6, 2016 b.

LAVADOS, Iván. La acreditación en Chile desde la perspectiva del Consejo Superior de Educación. En: PÉRSICO JIMÉNEZ, Pablo; PÉRSICO JIMÉNEZ, María Cecilia (Eds.). Acreditación de instituciones autónomas de educación superior. Santiago, Chile: Corporación de Promoción Universitaria (CPU), 1998. p. 11-18.

LEMAITRE, María José. Las atribuciones del Consejo Superior de Educación y el desarrollo de la regulación en Chile. Revista Chilena de Derecho, Chile, v. 24, n. 1, p. 39-47, 1997.

LEVY, Daniel C. El gobierno de los sistemas de educación superior: alternativas a la coordinación central. En: COX, C. (Ed.), Formas de gobierno en la educación superior: nuevas perspectivas. Santiago, Chile: FLACSO, 1990, p. 269-298.

LUCERO, Luis; THIERS, Sergio. Autoevaluación y acreditación. Visión de la Universidad Central. En: PERSICO, María Cecilia; PERSICO, Pablo (Eds.). Acreditación de instituciones autónomas de educación superior. Santiago, Chile: Corporación de Promoción Universitaria, 1998. p. 19-34.

LUHMANN, Niklas; SCHORR, Karl-Eberhard. Problems of reflection in the system of education. Neuwirth Münster y New York: Waxmann, 2000.

MARGINSON, Simon. Competition and Markets in Higher Education: a 'glonacal' analysis. Policy Futures in Education, United States, v. 2, n. 2, p. 175-244, 2004.

MARGINSON, Simon; RHOADES, Gary. Beyond national states, markets, and systems of higher education: A glonacal agency heuristic. Higher Education, United States, n. 43, p. 281-309, 2002.

MARTINIC, Sergio et al. Informe final. Sistematización y análisis de diálogos temáticos del plan de participación ciudadana para la reforma educacional. Santiago, Chile: Unidad de Inclusión y Participación Ciudadana. Ministerio de Educación de Chile, 2014 
De qué hablamos cuando hablamos de universidades. El Estado chileno y la idea contemporánea de la universidad

NEAVE, Guy. Patterns. En: RÜEGG, Walter (Ed.). A History of the university in Europa Universities since 1945. Cambridge Cambridge University Press, 2011. v. 4, p. 31-72.

PUSSER, Brian. Reconsidering higher education and the public good: the role of public spheres. En: TIERNEY, William G. (Ed.). Governance and the Public Good. New York: State University of New York Press, 2006. p. 11-28.

READINGS, Bill. The university in ruins. Cambridge, Massachusetts, and London, England: Harvard University, 1996.

SALAZAR, José Miguel.; LEIHY, Peodair S. El manual invisible: tres décadas de políticas de educación superior en Chile (1980-2010). Archivos Analíticos de Políticas Educativas, the United States, v. 21, n. 34, 2013.

SCOTT, John C. The mission of the university: Medieval to Postmodern Transformations. The Journal of Higher Education, United States, v. 77, n. 1, p. 1-39, 2006.

SIT, Victoria. The erosion of the university as a public sphere. Education Canada, Canada, v. 48, n. 4, p. 30-33, 2010.

SLAUGHTER, Sheila; LESLIE, Larry L. Expanding and elaborating the concept of academic Capitalism. Organization, the Netherlands, v. 8, n. 2, p. 154-161, 2001.

STÖLTING, Erhard; SCHIMANK, Uwe (Eds.). Die Krise der Universitäten. Wiesbaden: VS Verlag für Sozialwissenschaften, 2001.

${ }^{1}$ Julio Labraña Vargas

Universität Witten/Herdecke | Fakultät für Kulturreflexion Witten | Alemanha. Contato: Julio.LabranaVargas@uni-wh.de ORCID (iD) https://orcid.org/0000-0003-2441-8260 